\title{
Effect of high-load and high-volume resistance exercise on the tensiomyographic twitch response of biceps brachii
}

\author{
Juan Manuel García-Manso a , Darío Rodríguez-Matoso a , Samuel Sarmiento a , Yves de Saa ${ }^{\text {, }}$ \\ Diana Vaamonde $^{\text {b,* }}$, David Rodríguez-Ruiz ${ }^{\text {a }}$, Marzo Edir Da Silva-Grigoletto ${ }^{c}$ \\ a Laboratorio de Análisis y planificación del entrenamiento deportivo, Physical Education Department, University of Las Palmas de Gran Canaria, Spain \\ ${ }^{\mathrm{b}}$ Morphological Sciences Department, School of Medicine, University of Cordoba, Spain \\ ${ }^{\mathrm{C}}$ Centro Andaluz de Medicina del Deporte, Cordoba, Spain
}

\section{A R T I C L E I N F O}

\section{Article history:}

Received 29 May 2011

Received in revised form 6 January 2012

Accepted 7 January 2012

\section{Keywords:}

Fatigue

Muscle performance

Tensiomyography

\begin{abstract}
A B S T R A C T
The purpose of the present study was to assess the ability of TMG in detecting mechanical fatigue induced by two different resistance exercises on biceps brachii: high-volume (HV), and high-load (HL). Sixteen healthy subjects (age $25.1 \pm 2.6$ years; body mass $79.9 \pm 8.9 \mathrm{~kg}$; height $179 \pm 7.4 \mathrm{~cm}$ ) performed arm-curl in two different protocols (HV: $8 \times 15 \times 10 \mathrm{~kg}$, HL: $5 \times 3 \times 30 \mathrm{~kg}$ ). Tensiomyography was used to assess muscle response to both exercise protocols. The contractile capacity of biceps brachii significantly varied by means of the effects of potentiation and fatigue mechanisms that take place at different exercise phases. The most significant changes correspond to values of maximum radial displacement of muscle belly $\left(D_{\mathrm{m}}\right)$, sustained contraction time $\left(T_{\mathrm{s}}\right)$, relaxation time $\left(T_{\mathrm{r}}\right)$, and contraction velocity $\left(V_{\mathrm{c}}\right)$. The behavior of these parameters is, in general, similar in both exercise protocols, but they show subtle differences among them. During the first set, in both protocols, values for $V_{\mathrm{c}}$ increase, along with a decrease in $T_{\mathrm{r}}, T_{\mathrm{s}}$, and $D_{\mathrm{m}}$ values. Fatigue onset was evident from changes in such parameters, with HL being the first in showing these mechanisms. Tensiomyography has been shown to be highly sensitive in detecting fatigue-induced changes.
\end{abstract}

(c) 2012 Elsevier Ltd. All rights reserved.

\section{Introduction}

It is well documented that, when performing repetitive contractions, muscles experience, due to a fatigue effect, changes at biochemical, systemic, and structural levels that compromise their activity. An early definition of fatigue emphasizes that this functional response of the organism means a state in which an incapability to maintain the required or expected force is observed (Edwards, 1981; Bigland-Ritchie et al., 1983). Therefore, this state may be a transient phenomenon, of multifactorial origin, caused by physical activity. A gradual decrease in the force capacity of muscles or the endpoint of a sustained activity is observed by exhaustion of contractile function. Muscles that are used intensively show a progressive decline of performance which largely recovers after a sufficient period of rest. Conversely, potentiation is the increased functional change observed in the mammalian striated muscle after a previous muscle activity (Rassier, 2000; Abbate et al., 2000).

The cause of the loss of force during muscle fatigue has been attributed to different mechanisms, ranging from the generation

\footnotetext{
* Corresponding author. Address: Avda. Menendez Pidal s/n, School of Medicine, University of Cordoba, Cordoba, Spain. Tel./fax: +34 957218259.

E-mail addresses: fivresearch@yahoo.com,cm1vamad@uco.es (D. Vaamonde).
}

of the central command to the interaction between contractile proteins. The site of impairment depends on the task being performed, on its magnitude and on its origin; it varies with type, duration and intensity of the performed activity (Bigland-Ritchie et al., 1983; Tesch et al., 1990; Enoka and Stuart, 1992; Sacco et al., 2000; Gandevia, 2001; Hunter et al., 2003). According to this principle of effectiveness, it may be expected that acute fatigue determines changes in muscle response with typical characteristics.

A large number of studies on muscle fatigue normally draws attention to changes at the electrical excitation level, also known as central or neural fatigue (Garland and Gossen, 2002; Amann and Dempsey, 2008), or changes at metabolic level, also known as muscle or peripheral fatigue (de Ruiter et al.,1999; Sejersted and Sjøgaard, 2000). Studies analyzing effects of fatigue on muscle mechanical response are far sparser (Böl et al. 2011; Marini and Veicsteinas, 2010).

Tensiomyography (TMG), a non-invasive method for measuring the contractile properties of skeletal muscle, could probably offer an interesting alternative for analyzing the effects of fatigue on muscle response. Specific alterations in muscle mechanical response have been associated by means of this methodology with increases in maximum radial displacement of the muscle belly $\left(D_{\mathrm{m}}\right)$ (Šimunic et al., 2005; Smith and Hunter, 2006; García-Manso 
et al., 2011), sustained contraction time $\left(T_{\mathrm{s}}\right)$ (García-Manso et al., 2011), and relaxation time $\left(T_{\mathrm{r}}\right)$ (Rusu et al., 2009; García-Manso et al., 2011), as well as a decrease in contraction velocity $\left(V_{c}\right)$ (García-Manso et al., 2011). It is known that different exercise protocols elicit different muscle responses and fatigue which also involve different pathways (metabolic vs. neuromuscular); thus, we hypothesize that TMG is a highly sensitive tool to detect response changes in fatigued muscle, and, furthermore, to differentiate between different mechanisms in two strength exercise protocols.

Therefore, the aim of the present study was to assess the ability of TMG in detecting mechanical fatigue induced by two different resistance exercises on biceps brachii (BB).

\section{Methods}

\subsection{Participants}

Sixteen healthy, moderately active subjects (age $25.1 \pm$ 2.6 years; body mass $79.9 \pm 8.9 \mathrm{~kg}$; height $179 \pm 7.4 \mathrm{~cm}$ ), accustomed to strength training, volunteered to participate in the study. All subjects were informed of all details of the experimental procedures and the associated risks and discomforts. Each subject gave written informed consent forms previously approved by the Research Ethics Committee of the University of Las Palmas de Gran Canaria in line with the criteria of the Helsinki Declaration for research involving human beings.

\subsection{Study design}

Muscle response to two different resistance exercises was assessed in a cross-over design, in which participants performed both protocols in a randomized manner. The main dependent variables measured were the mechanical properties of muscle response such as maximum radial displacement of muscle belly $\left(D_{\mathrm{m}}\right)$, sustained contraction time $\left(T_{\mathrm{s}}\right)$, relaxation time $\left(T_{\mathrm{r}}\right)$, and contraction velocity $\left(V_{\mathrm{c}}\right)$. The independent variables were the two employed resistance exercise protocols. A one-week period was left as washout between both protocols.

\subsection{Procedures}

Biceps brachii contractile properties of dominant arm were assessed with the subject seated and the arm flexed $\left(90^{\circ}\right)$. For such purpose, a TMG device (TMG-BMC, Ljubljana, Slovenia) was used with application of a constant current electrical stimuli $(75 \mathrm{~mA})$. TMG is used to assess muscle stiffness (Pisot et al., 2008), estimate muscle composition (Dahmane et al., 2001, 2005; Simunic et al., 2011); additionally it is also used to assess contractile properties, and mechanical response of the superficial muscles under isometric conditions and electrical stimulation. The measuring point for each muscle was anatomically determined as a point of maximal muscle belly displacement detected with palpation during voluntary elbow extension (Dahmane et al., 2001; Šimunic et al., 2003; Valencic and Knez, 1997; Valencic et al., 2000, 2001). During measurements, the displacement-measuring sensor (GK40, Panoptik, Ljubljana, Slovenia) was pressed above the muscle belly perpendicularly to the muscle surface (Fig. 1). Self-adhesive bipolar electrodes (Compex Medical SA, Ecublens, Switzerland) were used and positioned $4 \mathrm{~cm}$ apart from midpoint. The robustness of the tool and the reproducibility of the method has been assessed in different studies (Dahmane et al., 2001; Krizaj et al., 2008; RodríguezMatoso et al., 2010; Šimunic et al., 2003; Šimunic and Valencic, 2001; Tous-Fajardo et al., 2010).

Muscle belly displacement (enlargement) during contraction (muscle belly radial displacement) takes place in the muscle belly

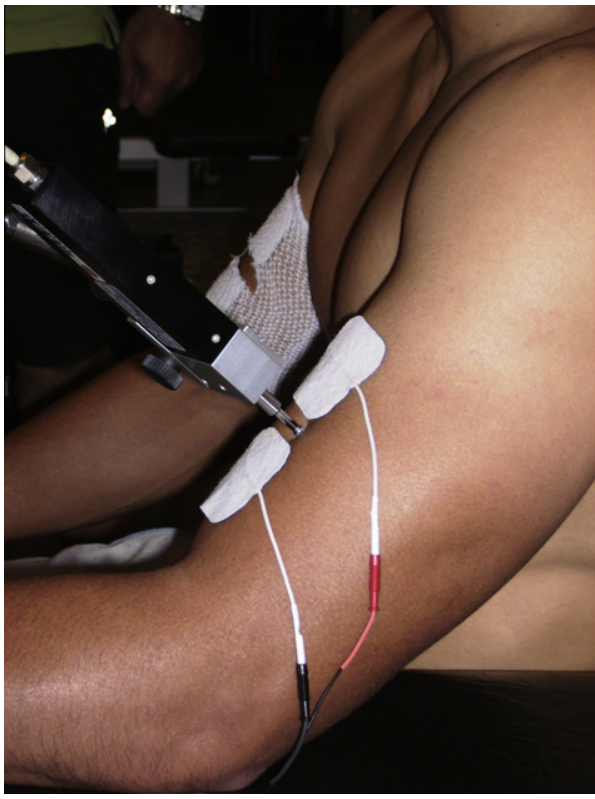

Fig. 1. Positioning of the TMG measuring system.

when a contraction is produced, under isometric conditions, as a result of an external electrical stimulus. In the present study, we analyzed four parameters, obtained from the magnitude of radial displacement of transverse muscle fibers and from the moment in which the displacements are produced.

\subsubsection{Analyzed parameters}

Maximum radial displacement $\left(D_{\mathrm{m}}\right)$ : determined by the radial displacement of muscle belly expressed in millimeters; it assesses muscle stiffness.

Sustained contraction time $\left(T_{\mathrm{s}}\right)$ : the theoretical time that contraction is maintained, and is calculated by determining the time period in which muscle response remains greater than $50 \% D_{\mathrm{m}}$.

Relaxation time $\left(T_{\mathrm{r}}\right)$ : the time in which muscle response decreases from $90 \%$ to $50 \% D_{\mathrm{m}}$.

The above mentioned parameters are shown in Fig. 2.

Contraction velocity $\left(V_{c}\right)$ : the mean contraction velocity is calculated when $D_{\mathrm{m}}$ reaches a $2-\mathrm{mm}$ value $\left(\Delta D_{\mathrm{m}} / d t\right)$; in brief, $D_{\mathrm{m}}$ was firstly graphed against $t, V_{\mathrm{c}}$ was calculated from the value of $D_{\mathrm{m}}$ and the time lapsed for such interval of muscle radial displacement. $V_{\mathrm{c}}$ was determined from a displacement of $D_{\mathrm{m}}$ that was equivalent to $2 \mathrm{~mm}\left(\Delta D_{\mathrm{m}} / t\right)$. To ensure a $2-\mathrm{mm}$ displacement,

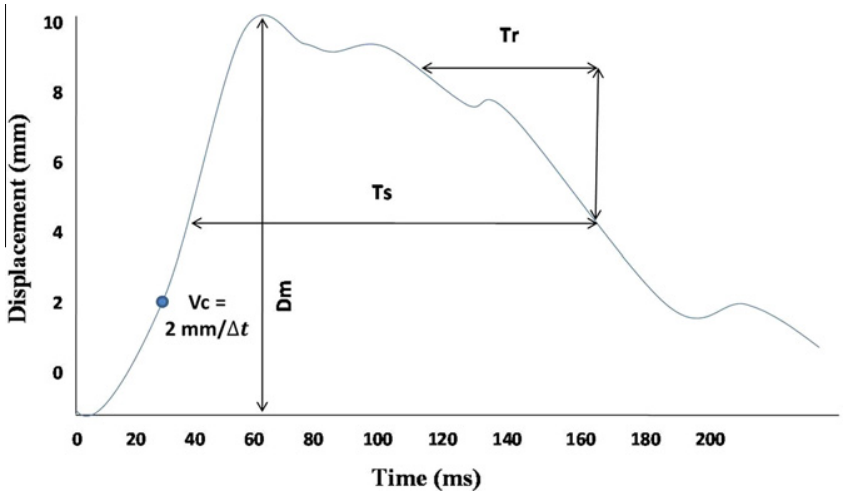

Fig. 2. Graph of typical signals of the muscle response to an electric stimulus by means of TMG. 


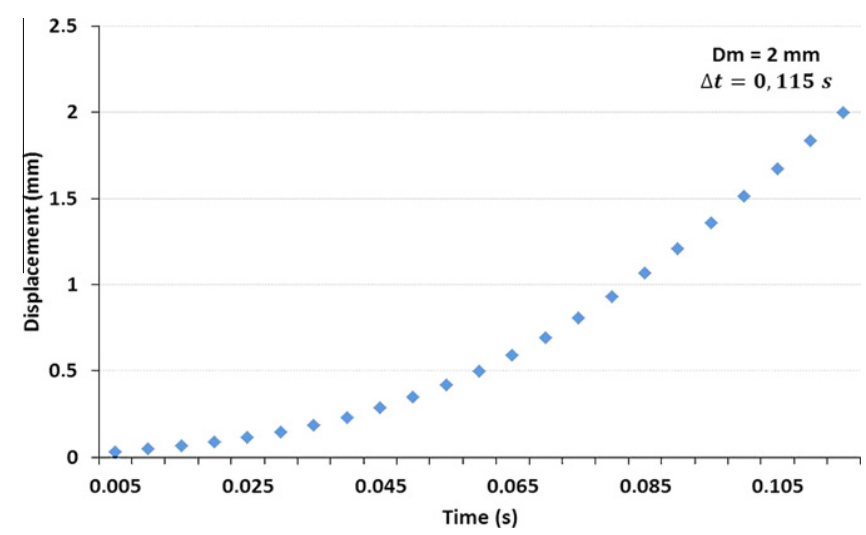

Fig. 3. Interpolation of $D_{\mathrm{m}}$ and $t$ for calculation of $V_{\mathrm{c}}$.

when recorded values were below or over $2 \mathrm{~mm}$, an interpolation was performed for $D_{\mathrm{m}}$ and $t$ (Fig. 3).

\subsubsection{Protocols}

Two different resistance exercise protocols were tested: highvolume (HV), and high-load (HL) with a BB curl exercise with bar. All exercise sessions were supervised by trained study personnel. For the exercise, subjects started from a standing up position with their torso upright while holding a barbell at a shoulderwidth grip; the palm of the hands were facing forward and the elbows kept close to the torso. Afterwards, while holding the upper arms stationary, the subjects curled the weights forward while contracting the biceps brachii and exhaling. Subjects were instructed to only move the forearms. The movement continued until biceps brachii was fully contracted and the bar was at shoulder level. Afterwards, subjects slowly began to bring the bar back to starting position while breathing in. The total time for the movement was three seconds: $1.5 \mathrm{~s}$ for the concentric phase and $1.5 \mathrm{~s}$ for the eccentric phase. Differences between both protocols were in workload, number of sets, and number of repetitions per set. The first protocol (HV) consisted in performing, with a controlled pace, eight sets with 15 repetitions each, recovery time between sets of one minute and a $10-\mathrm{kg}$ load $(8 \times 15 \times 10 \mathrm{~kg} \mathrm{rec}$. $1 \mathrm{~min})$. The second protocol (HL) consisted in performing, also with a controlled pace, five sets with three repetitions each, recovery time between sets of one minute, and a $30-\mathrm{kg}$ load $(5 \times 3 \times 30 \mathrm{~kg} \mathrm{rec}$. $1 \mathrm{~min})$.

During both exercise protocols, the first measurement took place in a rest situation (before the first set and without previous warm-up). The following measurement was performed $30 \mathrm{~s}$ after completing each set, putting the displacement sensor at the same point of the previous measurement. For such purpose, during the first assessment, a dermatographic pen was used to fix the measurement point. After the five (HL) and eight (HV) sets and their respective assessments, four more measurements were taken at $3,6,10$, and 15 min post-exercise.

\subsection{Statistics}

Traditional statistical methods were used in order to calculate both means and standard error of the mean (S.E.M.). The normality of the samples was calculated according to the Kolmogorov-Smirnov test. The effect of the different interventions: HL or HV protocols (independent variables) on $D_{\mathrm{m}}, T_{\mathrm{r}}, T_{\mathrm{s}}$ and $V_{\mathrm{c}}$ (dependent variables) was analyzed by means of analysis of variance (ANOVA) with repeated measurement of two factors (within): group and time. A Sidak correction was used to adjust the $P$-value in relation to the number of contrasts that were performed. A $P \leqslant 0.05$ crite- rion was used to establish statistical significance; for all the statistical tests the SPSS version v17 (SPSS Inc., Chicago, IL., USA) package for Windows was used.

\section{Results}

The behavior of $D_{\mathrm{m}}$ and $V_{\mathrm{c}}$ is shown in Figs. 4 and 5 while that of $T_{\mathrm{s}}$ and $T_{\mathrm{r}}$ is shown in Figs. 6 and 7.

From the first set on, a statistically significant decrease in $D_{\mathrm{m}}$ values in both protocols was observed with regards to rest values (HV: $20.9 \%$ decrease; HL: $17.4 \%$ decrease). This meant an increase in muscle stiffness due to a task effect. Such increase in stiffness continued throughout the remaining sets in $\mathrm{HV}$, reaching statistical significance ( $p=0.004$, time effect); with regards to HL a significant decrease was observed during the first three sets $(p=0.004$, time effect); from the 4 th set on the response was stabilized, and no statistically significant differences were observed. With regards to recovery, it is noteworthy that it was faster, albeit incomplete, in $\mathrm{HV}$. At the end of recovery ( $\left.\operatorname{Rec} 15^{\prime}\right), D_{\mathrm{m}}$ values in HL still showed significant differences with regards resting values $(p=0.021$, see Fig. 4).

The mechanical changes shown by the behavior of $D_{\mathrm{m}}$ are accompanied by similar behavior of $T_{\mathrm{s}}$ and $T_{\mathrm{r}}$, and also by an inverse response of $V_{c}$. Slight differences, however, were observed between each resistance exercise protocol. $T_{\mathrm{r}}$ significantly decreased in HV ( $p=0.001$, time effect); although there was a slight increase during the 5th and 6th sets trying to revert the situation, there was a further statistically significant decrease during the last two sets of the exercise; on the other hand, for HL, there was a statistically significant decrease until the 3rd set followed by an increase during the 4 th and 5 th sets (see Fig. 6 ). $T_{\mathrm{s}}$ significantly decreased in HV $(8 \times 15 \times 10 \mathrm{~kg})$ as the number of performed repetitions increased ( $p=0.002$, time effect), while, in HL $(5 \times 3 \times 30)$, the values stabilized during the last two sets (see Fig. 7).

On the contrary, $V_{c}$ increased significantly in both trainings ( $p=0.005$, time effect) showing similar behavior but with slight differences between them (Fig. 5 ). $V_{c}$ significantly increased from the first set to the third set, becoming stabilized on the 4th through 7th sets; after this latter set, a decrease was observed (see Fig. 5). $V_{c}$ increased in HL in a statistically significant manner up to the 4 th set to later decrease during the last set.

\section{Discussion}

We have studied the influence of HV and HL repetitive dynamic contractions on the human biceps brachii through two different resistance exercise protocols. The muscle response observed was an initial potentiation followed by a slight fatigue. Yet, the resistance exercise models employed present slight variations with regards to the changes that fatigue provokes in muscle response. Additionally, TMG has been shown to be a valid and non-invasive technique to evaluate the muscular response during resistance exercise.

While the selected exercise protocols for this study did not entail severe fatigue for the subjects, during the last set in both protocols, and especially in the HL work, symptoms of the appearance of acute fatigue that affect muscle response are observed. On the other hand, it seems that the muscle response was more efficient at the end of the first set.

During muscular contraction, the magnitude of neuromuscular activation is regulated via central descending pathways and also through sensory reflex pathways, including group Ib afferents from Golgi organs in the muscle-tendon complex and group Ia and group II afferents from muscle spindles (Gordon, 1991). Force production is often increased after a brief period of muscle activity, 


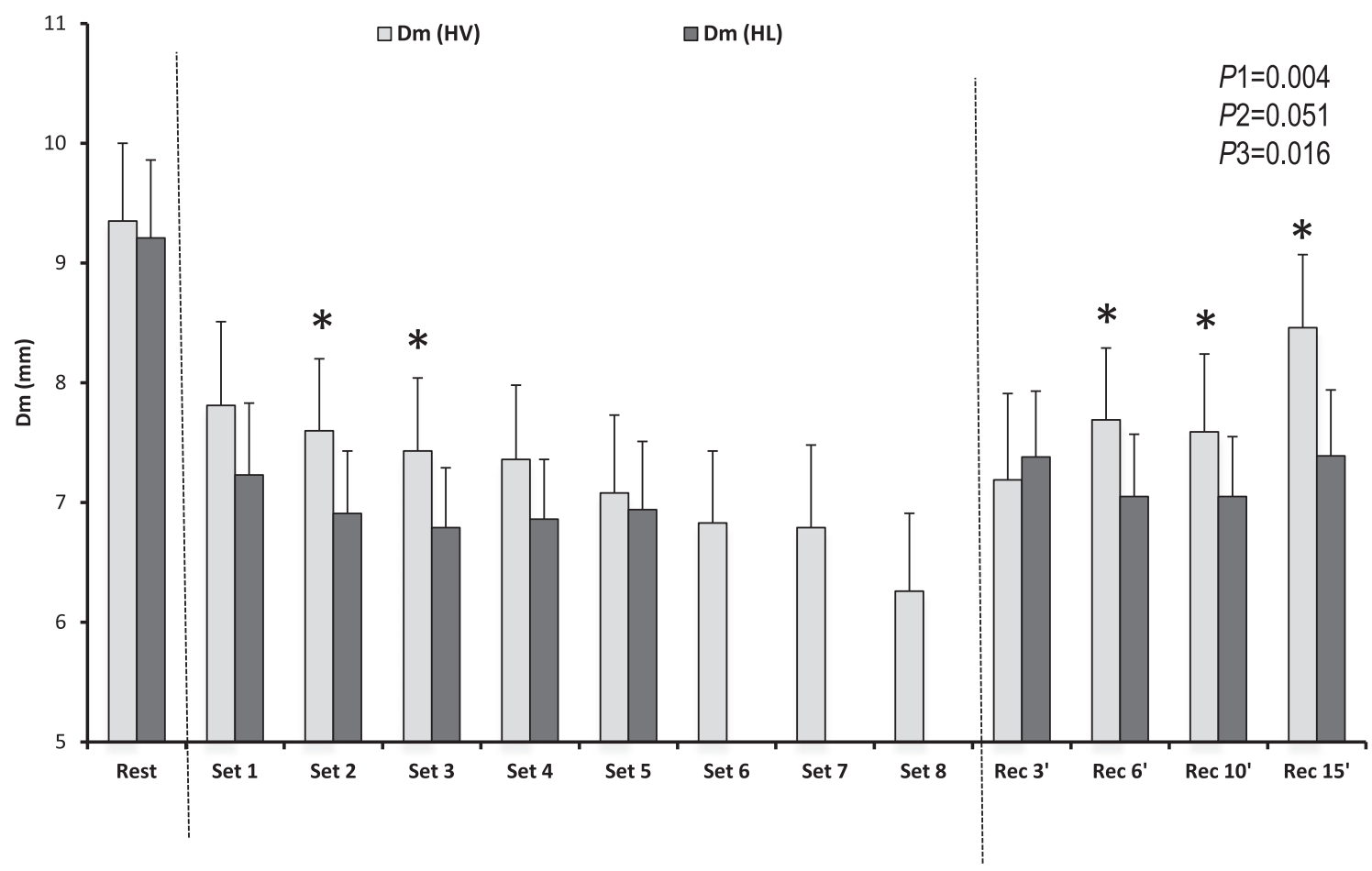

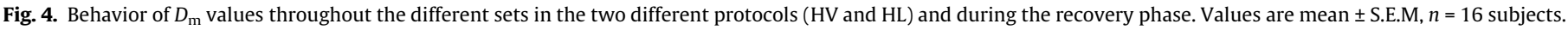

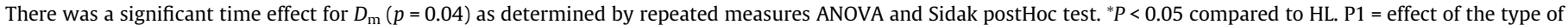
time; P2 = effect of the type of exercise protocol; P3 = effect of the type of time $\times$ exercise protocol.

and this phenomenon is called post-activation potentiation (Fowles and Green, 2003; Skurvydas and Zachovajevas, 1998). In the present case, as a result of the strength exercises used, all seems to indicate that post-activation potentiation was responsible for the observed changes in muscle response at the end of the first set. This mechanism becomes significant with changes in $V_{c}$ (increase), $D_{\mathrm{m}}$ (decrease), $T_{\mathrm{s}}$ (decrease), and $T_{\mathrm{r}}$ (decrease).

There is a correlation between this force potentiation and phosphorylation of the myosin regulatory light chain (Sweeney et al., 1993). During muscle activity, after each action potential, $\mathrm{Ca}^{2+}$ released forms the calcium/calmodulin complex, activating the kinase enzyme of the myosin light chains (Manning and Stull, 1982; Persechini et al.,1985; Sweeney et al., 1993). This enzyme enables the estate of phosphorylation of the myosin light chains, yet the phosphorylation-dephosphorylation cycle taking place during each muscle activation is relatively slow. This phenomenon may imply that the return to a rest state may be delayed for several minutes after finishing the contraction. Post-activation potentiation is generally larger in fast-twitch than in slow-twitch fibers (Sweeney et al., 1993).

Changes in phosphorylation of the myosin regulatory light chain, as well as alterations in muscle viscoelasticity, determine structural alterations in muscle that are manifested as an increase in its stiffness. On one hand, $D_{\mathrm{m}}$ represents muscle radial displacement, and indirectly evaluates muscle stiffness; on the other, it varies among subjects depending on how each muscle group has been trained. Therefore, we may think that $D_{\mathrm{m}}$ 's behavior in response to strength exercise will vary depending on the workload, recovery time between repetitions, and the type of contraction performed. Low $D_{\mathrm{m}}$ values indicate a high muscle tone and excessive stiffness in the muscle structures. On the other hand, elevated values indicate a lack of muscle tone or the appearance of muscle fatigue (Dahmane et al., 2001; Krizaj et al., 2008; Valencic et al., 2001). Fatigue becomes detectable when the observed changes start inverting with regards to the behavior observed during the first exercise set. Important levels of fatigue imply decreases in $V_{\mathrm{c}}$ and increases in the other three parameters $\left(D_{\mathrm{m}}, T_{\mathrm{s}}\right.$, and $\left.T_{\mathrm{r}}\right)$.

In recent years accumulating evidence has implicated altered intracellular $\mathrm{Ca}^{2+}$ regulation as a major contributor to muscle fatigue as sarcoplasmic reticulum $\mathrm{Ca}^{2+}$ release rate was markedly reduced after voluntary fatiguing contractions. This mechanism could likely be affected by sarcoplasmic reticulum $\mathrm{Ca}^{2+}$ release and all the $\mathrm{Ca}^{2+}$ buffers in the cell (Allen et al., 2008; Baylor and Hollingworth, 1998; Li and Handschumacher, 2002). This fatigue is partly associated to the $\mathrm{Na}^{+}-\mathrm{K}^{+}$pump regulation and to the changes it entails in intracellular and extracellular $\mathrm{Na}^{+}$and $\mathrm{K}^{+}$concentrations as shown by different studies (Clausen, 2003; Kabbara et al., 2000; Sjøgaard et al., 1985). These changes in the $\mathrm{Na}^{+}-\mathrm{K}^{+}$ pump affect sarcolemma and $\mathrm{T}$ tubules depolarization decreasing the release of $\mathrm{Ca}^{2+}$ and altering the muscle response.

In the HV protocol, the described processes were observed in a less intensive way, especially during the first moments, than in the HL protocol. The reason for this phenomenon may be the magnitude of the loads in each case. Nevertheless, at the end of the sets, symptoms of intense fatigue could also be observed in the HV protocol; such symptoms were detected by changes in the tendencies observed in the analyzed parameters $\left(V_{\mathrm{c}}, D_{\mathrm{m}}, T_{\mathrm{s}}\right.$, and $\left.T_{\mathrm{r}}\right)$. These changes vary, in magnitude and moment of appearance, according to the physical level of each subject.

With greater volume (120 repetitions) and lower load (10 kg) training, a major activation of the metabolic pathways used to supply ATP occurs, and is accompanied by large increases in the accumulation of metabolic by-products which may be the main trigger of muscle fatigue (Allen et al., 1995; Cady et al., 1989; Dawson et al., 1980; Fitts, 1994). In these cases, when the metaboreceptors are activated, there is inhibition of the motoneuron pool via a reflex pathway mediated by small-diameter group III and IV muscle afferents (Bangsbo, 1996; Sinoway et al., 1993). This response, 


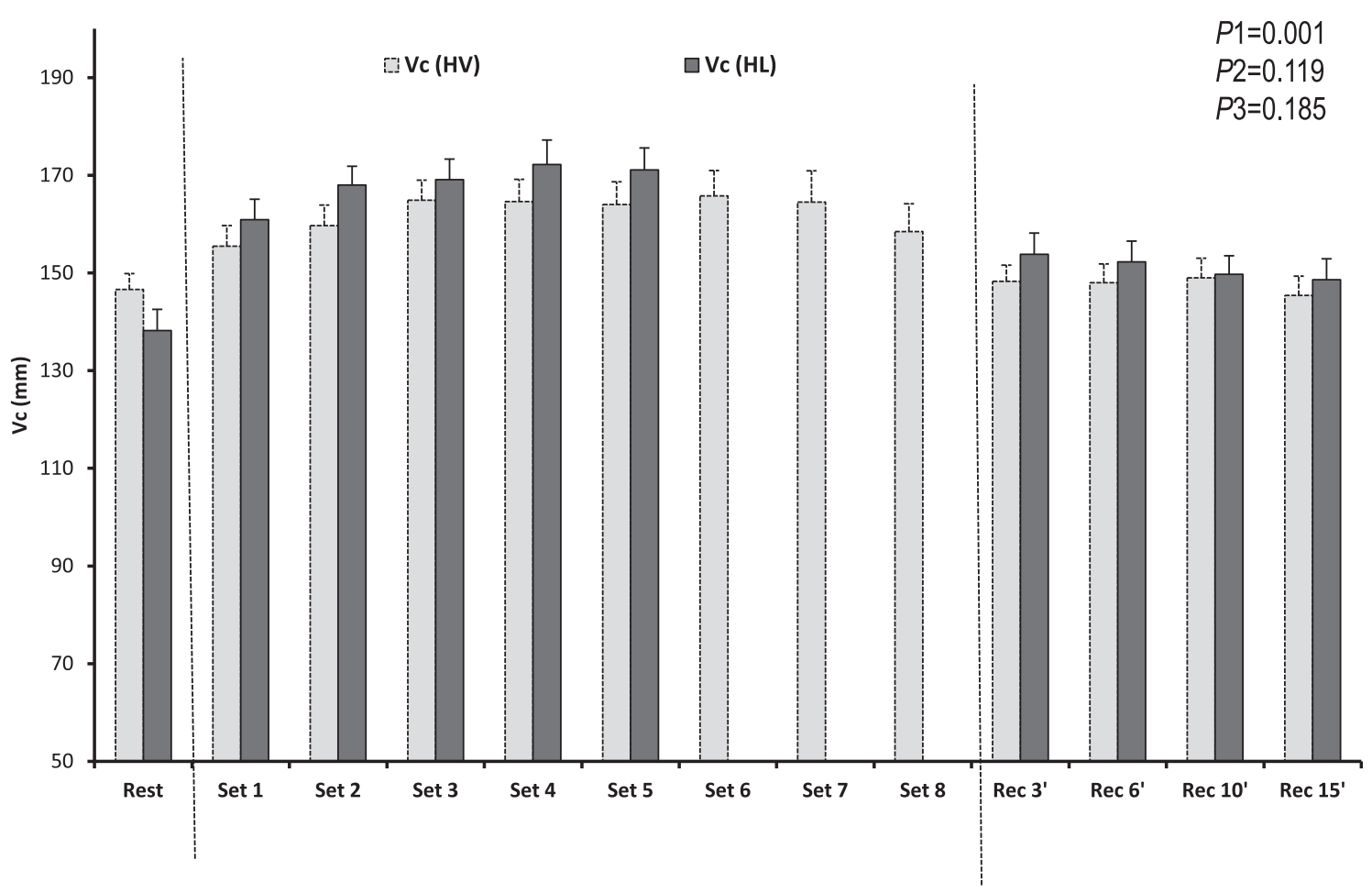

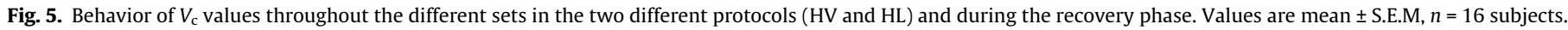

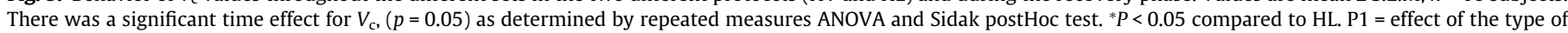
time; P2 = effect of the type of exercise protocol; P3 = effect of the type of time $\times$ exercise protocol.

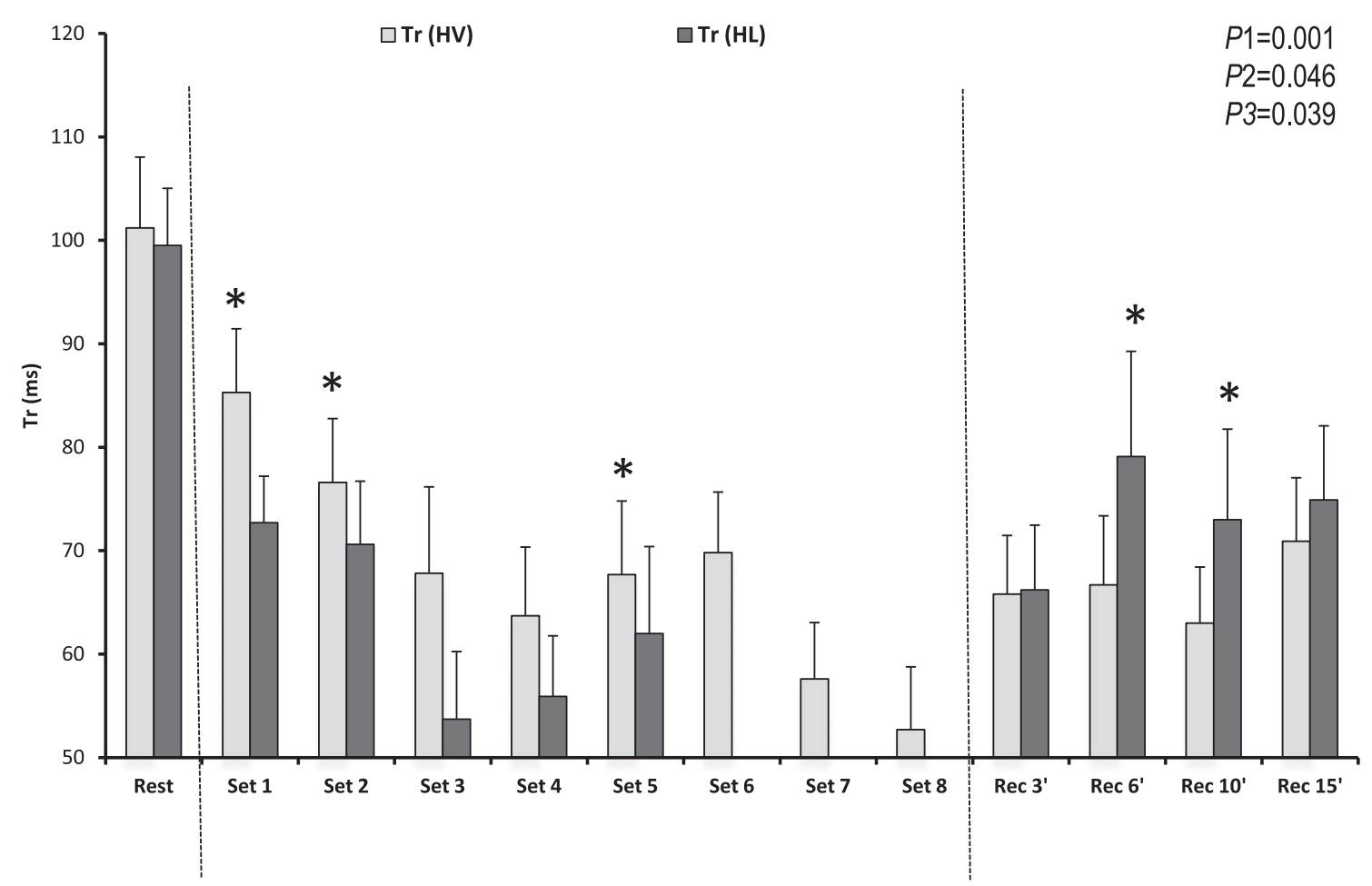

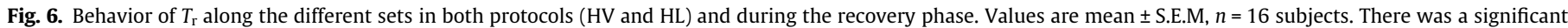

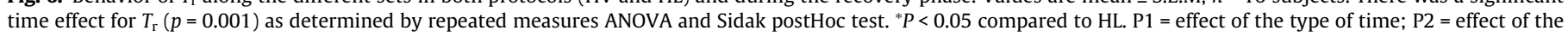
type of exercise protocol; P3 $=$ effect of the type of time $\times$ exercise protocol.

most likely, also affects the reduction in voluntary drive through spinal and supraspinal actions (Duchateau et al., 2002; Gandevia, 2001).
To the changes provoked by fatigue on the action potential and the myosin regulatory light chain activation, the alterations suffered by the fiber in the muscle relaxation mechanisms should 


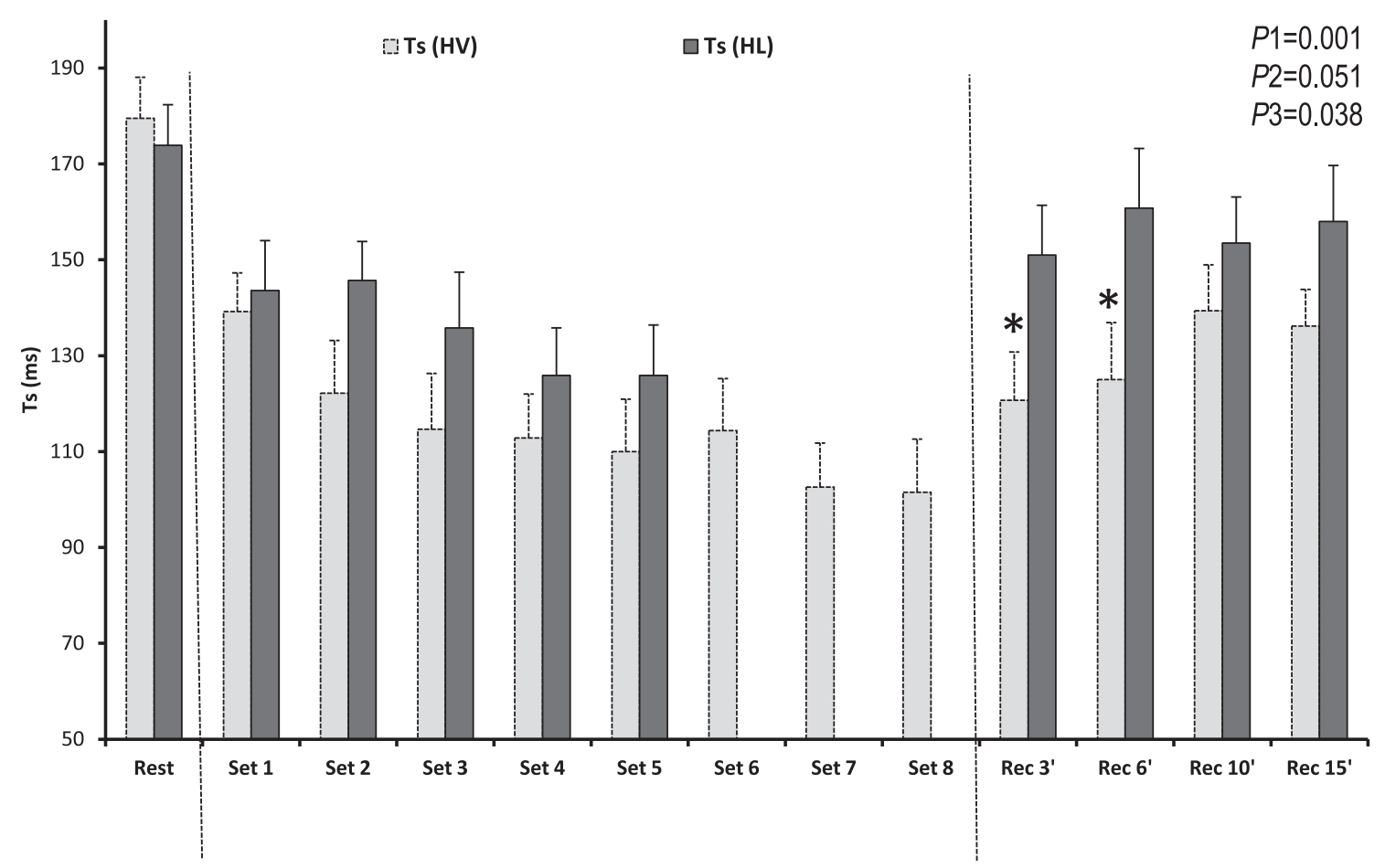

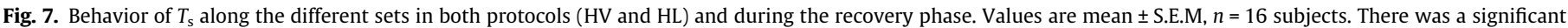

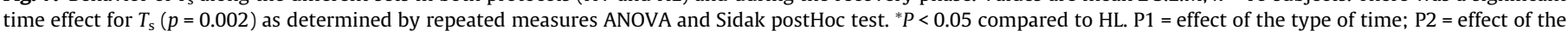
type of exercise protocol; P3 $=$ effect of the type of time $\times$ exercise protocol.

be added. Relaxation of skeletal muscle cells is a complex process that involves changes in SR $\mathrm{Ca}^{2+}$ handling and cross-bridge function (Allen et al., 2008). Skeletal muscle fatigue is generally accompanied by a progressive slowing of relaxation, affecting the force-generating potential. This situation is especially observed when the muscle works repeatedly with short recovery times between each movement such as what happened in the used protocols.

In none of the protocols a complete recovery of $T_{\mathrm{s}}$ and $T_{\mathrm{r}}$ values was achieved during the $15^{\prime}$ after termination of the exercise (Fig. 3 ). $T_{\mathrm{s}}$ recovery was $75.9 \%$ in $\mathrm{HV}$, and $90.8 \%$ in $\mathrm{HL} . T_{\mathrm{r}}$ recovery was less efficient, reaching only values of $70.1 \%$ and $75.2 \%$, respectively. The same happened with $D_{\mathrm{m}}$ (Fig. 2), where recovery was 90.4\% (HV) and $80.2 \%(\mathrm{HL})$. On the contrary, for $V_{c}$, in both cases (Fig. 2), recovery was complete $15^{\prime}$ after finishing the exercise. Nonetheless, recovery was faster in HV, where values close to normality were achieved three minutes after finishing the task. This situation indicates that $V_{\mathrm{c}}$ had a behavior similar to that detected when studying recovery of the motor nerve which, according to Béliveau et al., 1991, recovers initial values of mean impulse frequency and conduction velocity of the motor nerve just few minutes after performing a resistance exercise.

In summary, it can be observed how the manifestation of the contractile capacity of biceps brachii significantly varies due to the effects of potentiation and fatigue that exercise entails during the different phases. Fatigue may be detected by means of the analysis that TMG provides us, especially the values of $D_{\mathrm{m}}, V_{\mathrm{c}}, T_{\mathrm{s}}$, and $T_{\mathrm{r}}$. These parameters reflect the alterations that take place in the muscle at the structural and neural levels. The behavior in these parameters is, in general, similar in both used exercise models (HV and $\mathrm{HL}$ ), yet showing subtle differences between them. In both cases, $V_{\mathrm{c}}$ increased during the first set while $D_{\mathrm{m}}, T_{\mathrm{s}}$, and $T_{\mathrm{r}}$ decreased. When fatigue starts appearing, these behaviors became inverted, with the high-load work being the first in showing these mechanisms.

\section{References}

Abbate F, Sargeant AJ, Verdijk PWL, de Haan A. Effects of hightfrecuency initial pulses and posttetanic potentiation on power output of skeletal muscle. J Appl Physiol 2000;88:35-40.

Allen DG, Lännergren J, Westerblad $\mathrm{H}$. Muscle cell function during prolonged activity: cellular mechanisms of fatigue. Exp Physiol 1995;80:497-527.

Allen DG, Lamb GD, Westerblad H. Skeletal muscle fatigue: cellular mechanisms. Physiol Rev 2008;88:287-332.

Amann M, Dempsey JA. Locomotor muscle fatigue modifies central motor drive in healthy humans and imposes a limitation to exercise. J Physiol 2008;586:161-73.

Bangsbo J. Physiological factors associated with efficiency in high intensity exercise. Sports Med 1996;22:299-305.

Baylor SM, Hollingworth S. Model of sarcomeric Ca2+ movements, including ATP $\mathrm{Ca} 2+$ binding and diffusion, during activation of frog skeletal muscle. J Gen Physiol 1998;112:297-316.

Béliveau L, Helal JN, Gaillard E, Van Hoecke J, Atlan G, Bouissou P. EMG spectral shift and 31P-NMR-determined intracellular $\mathrm{pH}$ in fatigued human biceps brachii muscle. Neurology 1991;41:1998-2001.

Bigland-Ritchie B, Johansson R, Lippold OC, Smith S, Woods JJ. Changes in motoneuron firing rates during sustained maximal voluntary contractions. J Physiol 1983;340:335-46.

Böl M, Stark H, Schilling N. On a phenomenological model for fatigue effects in skeletal muscles. J Theor Biol 2011;281:122-32.

Cady EB, Jones DA, Lynn J, Newham DJ. Changes in force and intracellular metabolites during fatigue of human skeletal muscle. J Physiol 1989;418: 311-25.

Clausen $\mathrm{T}$. Na+-K+ pump regulation and skeletal muscle contractility. Physiol Rev 2003;83:1269-324.

Dahmane R, Valencic V, Knez N, Erzen I. Evaluation of the ability to make noninvasive estimation of muscle contractile properties on the basis of the muscle belly response. Med Biol Eng Comput 2001;39:51-5.

Dahmane R, Djordjevic S, Simunic B, Valencic V. Spatial fiber type distribution in normal human muscle histochemical and tensiomyographical evaluation. J Biomech 2005;38:2451-9.

Dawson RM, Hemington N, Irvine RF. The inhibition and activation of $\mathrm{Ca} 2+$ dependent phosphatidylinositol phosphodiesterase by phospholipids and blood plasma. Eur J Biochem 1980;112:33-8.

de Ruiter CJ, Jones DA, Sargeant AJ, de Haan A. Temperature effect on the rates of isometric force development and relaxation in the fresh and fatigued human adductor pollicis muscle. Exp Physiol 1999;84:1137-50.

Duchateau J, Balestra C, Carpentier A, Hainaut K. Reflex regulation during sustained and intermittent submaximal contractions in humans. J Physiol 2002;541:959-67. 
Edwards RH. Human muscle function and fatigue. Ciba Found Symp 1981;82:1-18. Enoka RM, Stuart DG. Neurobiology of muscle fatigue. J Appl Physiol 1992:72:1631-48.

Fitts RH. Cellular mechanisms of muscle fatigue. Physiol Rev 1994;74:49-94.

Fowles JR, Green HJ. Coexistence of potentiation and low-frequency fatigue during voluntary exercise in human skeletal muscle. Can J Physiol Pharmacol 2003;81:1092-100.

Gandevia SC. Spinal and supraspinal factors in human muscle fatigue. Physiol Rev 2001;81:1725-89.

García-Manso JM, Rodríguez-Ruiz D, Rodríguez-Matoso D, de Saá Y, Sarmiento S, Quiroga ME. Assessment of muscle fatigue after an ultraendurance triathlon using Tensiomyography (TMG). J Sport Sci 2011;29:619-25.

Garland SJ, Gossen ER. The muscular wisdom hypothesis in human muscle fatigue. Exerc Sport Sci Rev 2002;30:45-9.

Gordon J. Spinal mechanisms of motor coordination. In: Kandel ER, Schwartz JH, Jessell TM, editors. Principles of neural science (3rd ed.). Englewood Cliffs, NJ: Prentice-Hall; 1991. p. 581-95.

Hunter SK, Lepers R, MacGillis CJ, Enoka RM. Activation among the elbow flexor muscles differs when maintaining arm position during a fatiguing contraction. J Appl Physiol 2003;94:2439-47.

Kabbara AA, Nguyen LT, Stephenson GMM, Allen DG. Intracellular calcium during fatigue of cane toad skeletal muscle in the absence of glucose. J Muscle Res Cell Motil 2000;21:481-9.

Krizaj D, Simunic B, Zagar T. Short-term repeatability of parameters extracted from radial displacement of muscle belly. J Electromyogr Kinesiol 2008;18:645-51.

Li W, Handschumacher RE. Identification of two calcineurin B-binding proteins: tubulin and heat shock protein 60. Biochim Biophys Acta 2002;1599:72-81.

Manning DR, Stull JT. Myosin light chain phosphorylation-dephosphorylation in mammalian skeletal muscle. Am J Physiol Cell Physiol 1982;242:C234-41.

Marini M, Veicsteinas A. The exercised skeletal muscle: a review. Eur J Transl Myology 2010;20(3):105-20.

Persechini A, Stull JT, Cooke R. The effect of myosin phosphorylation on the contractile properties of skinned rabbit skeletal muscle fibers. J Biol Chem 1985;260:7951-4

Pisot R, Narici MV, Simunic B, De Boer M, Seynnes O, Jurdana M, et al. Whole muscle contractile parameters and thickness loss during 35-day bed-rest. Eur J Appl Physiol 2008;104(2):409-14.

Rassier DE. The effects of length on fatigue and twitch potentiation in human skeletal muscle. Clin Physiol 2000;20:474-82.

Rodríguez-Matoso D, Rodríguez-Ruiz D, Sarmiento S, Vaamonde D, Da SilvaGrigoletto ME, García-Manso JM. Reproducibility of muscle response measurements using tensiomyography in a range of positions. Rev Andal Med Deporte 2010;3:81-6.

Rusu L, Cernaianu S, Vasilescu M, Baltac G, Ciocanescu D, Fortan C. Assessment of knee stability using neuromuscular measurement in soccer players. XVIII International Congress on Sports Rehabilitation and Traumatology, Italy, 2009. p. 98-99.

Sacco P, Thickbroom GW, Byrnes ML, Mastaglia FL. Changes in corticomotor excitability after fatiguing muscle contractions. Muscle Nerve 2000;23:1840-6.

Sejersted OM, Sjøgaard G. Dynamics and consequences of potassium shifts in skeletal muscle and heart during exercise. Physiol Rev 2000;80:1411-81.

Šimunič B, Valenčič V. 2001. Non-invasive selective measurement of M. vastus medialis and M. vastus lateralis contractile properties at different knee angles. In: Proceedings of the tenth electrotechnical and computer science conference ERK, vol. B, Portorož, Slovenia, Ljubljana: IEEE Region 8, Slovenian Section IEEE; 24-26 September 2001. p. 363-66.

Šimunič B. 2003. Modelling of longitudinal and transversal skeletal muscle belly deformation. PhD Thesis. School of Electrical Engineering. Ljubljana (Slovenia). 2003.

Šimunič B., Rozman S, Pišot R. Detecting the velocity of the muscle contraction. In: III International symposium of new technologies in sports, Sarajevo: 2005.

Simunic B, Degens H, Rittweger J, Narici M, Mekjavic IB, Pisot R. Noninvasive estimation of myosin heavy chain composition in human skeletal muscle. Med Sci Sports Exerc 2011. doi:10.1249/MSS.0b013e31821522d0.

Sinoway LI, Hill JM, Pickar JG, Kaufman MP. Effects of contraction and lactic acid on the discharge of group III muscle afferents in cats. J Neurophysiol 1993;69:1053-9.

Sjøgaard G, Adams RP, Saltin B. Water and ion shifts in skeletal muscle of humans with intense dynamic knee extension. Am J Physiol Regul Integr Comp Physiol 1985;248:R190-6

Skurvydas A, Zachovajevas P. Is post-tetanic potentiation, low frequency fatigue (LFF) and pre-contractile depression (PCD) coexistent in intermittent isometric exercises of maximal intensity? Acta Physiol Scand 1998;164:127-33.

Smith IJ, Hunter AM. The Effect of titanic stimulated induced fatigue on the relationship between TMG and force production of the gastrocnemius medialis. Med Sci Sports Exerc 2006;38(5):S179-80.

Sweeney HL, Bowman BF, Stull JT. Myosin light chain phosphorylation in vertebrate striated muscle: regulation and function. Am J Physiol Cell Physiol 1993;264:C1085-95.

Tesch PA, Dudley GA, Duvoisin MR, Hather BM, Harris RT. Force and EMG signal patterns during repeated bouts of concentric or eccentric muscle actions. Acta Physiol Scand 1990;138:263-71.
Tous-Fajardo J, Moras G, Rodríguez-Jiménez S, Usach R, Doutres DM, Maffiuletti NA Inter-rater reliability of muscle contractile property measurements using noninvasive tensiomyography. J Electromyogr Kinesiol 2010;20:761-6.

Valencic V, Knez N. Measuring of skeletal muscles dynamic properties. Artif Organs $1997 ; 21: 240-2$

Valencic V, Djordjevic S, Knez N, Dahmane R, Coh M, Jurcic-Zlobec B, et al. Contractile properties of skeletal muscles detection by tensiomiographic measurement method. 2000 Pre-Olympic Congress, Brisbane, Australia, 2000 p. 507.

Valencic V, Knez N, Simunic B. Tensiomyography: detection of skeletal muscle response by means of radial muscle belly displacement. Biomed Eng 2001;1:1-10

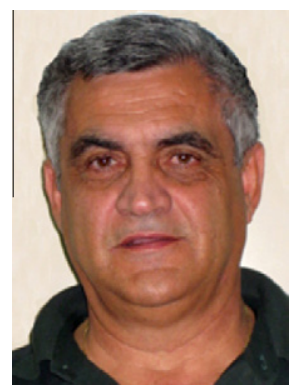

Juan Manuel Garcia-Manso, B.S., M.S., Ph.D. received his B.S. in Physical Activity and Sport Sciences from the Polytechnic University of Madrid, his M.S. in High Sports Performance from the Spanish Olympic Committee and his Ph.D. in Sports Sciences from the Polytechnic University of Madrid. He is a Professor at the University of Las Palmas de Gran Canariás Physical Education Department and directs the "Sports Training Analysis and Planning Laboratory (LAPED)". He has research interest in several areas of Sports Sciences including tensiomyography.

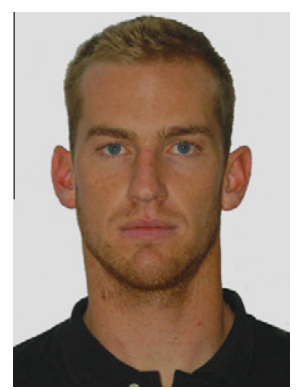

Dario Rodriguez Matoso, B.S. received his B.S. in Physical Activity and Sport Sciences from the University of Las Palmas de Gran Canaria in 2009. He is a researcher at the "Sports Training Analysis and Planning Laboratory (LAPED)" of the University of Las Palmas de Gran Canaria. His main area of interest is the use and applications of tensiomyography in different populations.

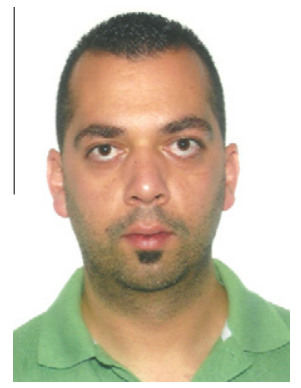

Samuel Sarmiento, B.S., Ph.D, received his B.S. in Physical Activity and Sport Sciences from the University of Las Palmas de Gran Canaria in 2004 and his Ph.D. in Physical Activity, Health and Sports Performance from the same institution in 2008. He is a post-doc researcher of the University of Las Palmas de Gran Canariás Physical Education Department since 2007. His main area of interest is the effect of physical activity on cognitive function of elderly people with Alzheimer-type deterioration.

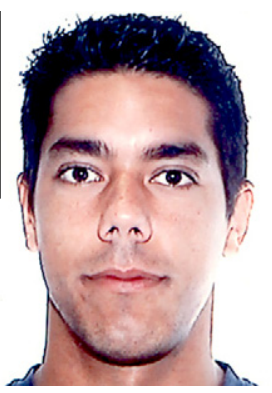

Yves de Saá, B.S received his B.S. in Physical Activity and Sport Sciences from the University of Las Palmas de Gran Canaria. Since 2006 he is a research fellow at the "Sports Training Analysis and Planning Laboratory (LAPED)" of the University of Las Palmas de Gran Canariás Physical Education Department. 


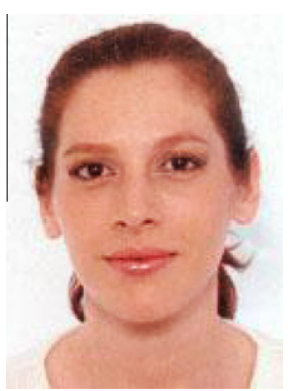

Diana Vaamonde, B.S., M.S., Ph.D. was born in the Canary Islands, Spain. She has received a B.S. Biology from Washington and Lee University in 1998 and her Ph.D. degree in Sport Sciences in 2006 from the University of Córdoba, Spain. She is Assistant Professor at the Morphological Sciences Department of the University of Cordobás Medical School. She has been director to several clinical laboratories and her research interest is the interaction of several biological systems as response to sports training. She has recently created the International Network on Physical Exercise and Fertility.

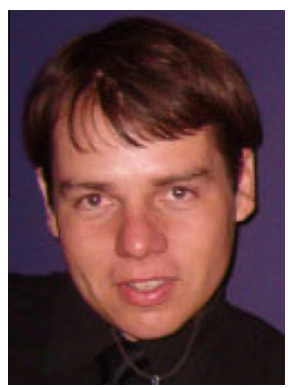

Marzo E. Da Silva-Grigoletto, B.S., M.S., Ph.D. was born in Brazil (RS). He received his Ph.D. degree in Sport Sciences in 2006 from the University of Córdoba, Spain. He is a M.S. in Research Methodology in Health Sciences. He has been strength and conditioning trainer to Spanish Superleague volleyball teams and now is an advisor to several athletes and teams. He now teaches at the Physical Education and Sports Department of the University of Seville, is Editor-in-Chief of the Andalusian Journal of Sports Medicine (Elsevier), President of the Scientific Sport Association, and is in charge of the $\mathrm{I}+\mathrm{D}+\mathrm{I}$ of the International Institute of Physical Exercise Science and Health.

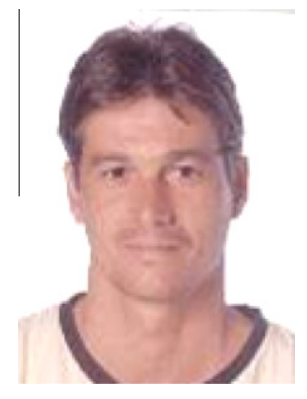

David Rodriguez Ruiz, B.S., Ph.D. received his B.S. in Physical Activity and Sport Sciences from the University of Las Palmas de Gran Canaria in 1995 and his Ph.D. in Physical Education from the same institution in 1999. He is Associate Professor of the University of Las Palmas de Gran Canariás Physical Education Department since 2007. He has been a researcher in the "Sports Training Analysis and Planning Laboratory (LAPED)" since 2008. 